PROCEEDINGS OF THE

AMERICAN MATHEMATICAL SOCIETY

Volume 140, Number 9, September 2012, Pages 3049-3054

S 0002-9939(2012)11163-3

Article electronically published on January 31, 2012

\title{
ON THE RADICAL OF A FREE MALCEV ALGEBRA
}

\author{
I. P. SHESTAKOV AND A. I. KORNEV
}

(Communicated by Kailash C. Misra)

\begin{abstract}
We prove that the prime radical $\operatorname{rad} \mathcal{M}$ of the free Malcev algebra $\mathcal{M}$ of rank more than two over a field of characteristic $\neq 2$ coincides with the set of all universally Engelian elements of $\mathcal{M}$. Moreover, let $T(\mathbb{M})$ be the ideal of $\mathcal{M}$ consisting of all stable identities of the split simple 7 -dimensional Malcev algebra $\mathbb{M}$ over $F$. It is proved that $\operatorname{rad} \mathcal{M}=J(\mathcal{M}) \cap T(\mathbb{M})$, where $J(\mathcal{M})$ is the Jacobian ideal of $\mathcal{M}$. Similar results were proved by I. Shestakov and E. Zelmanov for free alternative and free Jordan algebras.
\end{abstract}

An algebra $M$ is called a Malcev algebra if it satisfies the identities

$$
\begin{gathered}
x^{2}=0, \\
J(x, x y, z)=J(x, y, z) x,
\end{gathered}
$$

where $J(x, y, z)=(x y) z+(z x) y+(y z) x$ is the Jacobian of the elements $x, y, z$ [7, 9, 5. Since for a Lie algebra the Jacobian of any three elements vanishes, Lie algebras fall into the variety of Malcev algebras. Among the non-Lie Malcev algebras, the traceless elements of the octonion algebra with the product given by the commutator $[x, y]=x y-y x$ is one of the most important examples [9, 5, 6].

In 1977 I. P. Shestakov 11 proved that the free Malcev algebra $\mathcal{M}_{n}$ on $n \geq 9$ free generators is not semiprime; that is, $\mathcal{M}_{n}$ contains nonzero nilpotent ideals. In 1979, V. T. Filippov 3 extended this result to free Malcev algebras with more than four generators. Therefore, the prime radical $\operatorname{rad} \mathcal{M}_{n} \neq 0$ for $n>4$, and a natural question on the description of this radical arises.

For free alternative algebras, it was proved by Shestakov in 10 that the prime radical coincides with the set of nilpotent elements. A similar fact was established by E. Zel'manov [15] for free Jordan algebras.

In anticommutative algebras, the role of nilpotent elements is played by engelian elements. An element $a$ of an algebra $M$ is called engelian if the operator of right multiplication $R_{a}: x \mapsto x a$ is nilpotent. We will call an element $a \in M$ universally engelian if, for every algebra $M^{\prime} \supseteq M$, the element $a$ is engelian in $M^{\prime}$. In other words, the image $\mathcal{R}_{a}$ of the element $a$ in the (associative) universal multiplicative enveloping algebra $\mathcal{R}(M)$ of $M$ is nilpotent. In the present paper, we prove that the

Received by the editors February 23, 2011 and, in revised form, March 31, 2011.

2010 Mathematics Subject Classification. Primary 17D10, 17D05, 17A50, 17A65.

Key words and phrases. Malcev algebra, free algebra, prime radical, nilpotent element, Engelian element.

The first author was supported by FAPESP grant 2010/50347-9 and CNPq grant 305344/ 2009-9.

The second author was supported by FAPESP grant 2008/57680-5.

(C)2012 American Mathematical Society Reverts to public domain 28 years from publication 
prime radical of the free Malcev algebra over a field of characteristic $\neq 2$ coincides with the set of all universally engelian elements.

We consider the algebras over a field $F$ of characteristic $\neq 2$. Denote $[x, y, z]=$ $(x y) z+x(y z)$ and $\{x, y, z\}=[x, y, z]-[x, z, y]=J(x, y, z)-3(y z) x$, and consider the function

$$
h(y, z, t, x)=\{y z, t, x\} x+\{y x, z, x\} t .
$$

The variety of Malcev algebras defined by the identity $h=0$ is denoted by $\mathcal{H}$.

An anticommutative algebra $A$ is called an algebra without zero divisors if for any $a, b \in A$ the equality $a b=0$ implies that $a, b$ are linearly dependent.

Below we sometimes will omit the parentheses in left-normed products; that is, $a b c d$ means $((a b) c) d$ and $a b^{k}$ means $a R_{b}^{k}$.

Lemma 1. Let $A$ be an algebra of dimension $\geq 2$ from the variety $\mathcal{H}$ without zero divisors. If $A$ has a nonzero Engelian element, then $A$ is isomorphic to sl(2) or to the nonabelian two-dimensional Lie algebra.

Proof. Let $f \in A, f \neq 0$ be an engelian element of the algebra $A$. The operator $R_{f}$ is nilpotent, and it is well known that $A$ is a direct sum of cyclic subspaces of operator $R_{f}$, i.e.

$$
A=\bigoplus_{i}\left(F a_{i}+F a_{i} R_{f}+\cdots+F a_{i} R_{f}^{n_{i}-1}\right),
$$

where $a_{i} R_{f}^{n_{i}}=0$. Since $\left(\bigoplus_{i} F a_{i} R_{f}^{n_{i}-1}\right) \cdot f=\bigoplus_{i}\left(F a_{i} R_{f}^{n_{i}}\right)=0$, by the absence of zero divisors we have the inclusion $\bigoplus_{i} F a_{i} R_{f}^{n_{i}-1} \subseteq F f$, and $A=F a+F a f+\cdots+$ $F a f^{n-1}$, where $a f^{n-1}=f$.

If $n=2$, then $A=F a+F f, a f=f$, and we have the nonabelian two-dimensional Lie algebra.

Let $n \geqslant 3$. Substituting $x=z=f$ in $h$, we obtain for any $y, t \in A$ the equality $(t f)(y f) f=0$. This implies $(a f)\left(a f^{n-1}\right) f=0$, i.e. $(a f)\left(a f^{n-1}\right) \in F f$. Therefore, $a f^{2}=(a f) f=(a f)\left(a f^{n-1}\right) \in F f$, and $n=3$. We can choose $a$ such that $a f^{2}=f$. Consider $a(a f)=\alpha a+\beta a f+\gamma a f^{2}$ for some $\alpha, \beta, \gamma \in F$. We have $0=J(a, a f, f)=$ $a(a f) f+a f f a=(\alpha-1) a f+\beta a f^{2}$. Since $a f$ and $a f^{2}$ are linearly independent, it follows that $\alpha=1$ and $\beta=0$, i.e. $a(a f)=a+\gamma a f^{2}$. Now setting $e_{1}=a+\frac{\gamma}{2} a f^{2}$ and $e_{2}=a f^{2}$ we have $e_{1} e_{2}=\left(a+\frac{\gamma}{2} a f^{2}\right) a f^{2}=a f . e_{1}\left(e_{1} e_{2}\right)=\left(a+\frac{\gamma}{2} a f^{2}\right)(a f)=$ $a($ af $)-\frac{\gamma}{2} a f^{2}=e_{1}$. Consequently, $A=F e_{1} \oplus F e_{2} \oplus F e_{3}$, where $e_{1} e_{2}=e_{3}, e_{1} e_{3}=e_{1}$, $e_{2} e_{3}=-e_{2}$, i.e. $A \cong s l(2)$. The lemma is proved.

Lemma 2. Let $\mathbb{O}$ be an octonion division algebra over a field $F$. Then the Malcev algebra sl $(\mathbb{O})$ of traceless octonions has no zero divisors.

Proof. Assume that $a, b \in \mathbb{O}, \operatorname{tr}(a)=\operatorname{tr}(b)=0$ and $[a, b]=0$. Recall that we have the following identity in $\mathbb{O}$ (see [16, identity (2.9)]):

$$
x y+y x=\operatorname{tr}(x) y+\operatorname{tr}(y) x+\operatorname{tr}(x y)-\operatorname{tr}(x) \operatorname{tr}(y) .
$$

Therefore, we have $0 \neq 2 a b=\operatorname{tr}(a b) \in F$ and hence $b \in F a^{-1}$. On the other hand, $0 \neq a^{2}=-n(a) \in F$; hence $a^{-1} \in F a$. Therefore, $a$ and $b$ are linearly dependent.

Corollary 1. Let $\mathbb{O}$ be an octonion division algebra over a field $F$. Then the Malcev algebra $\operatorname{sl}(\mathbb{O})$ of traceless octonions has no nonzero engelian elements. 
Proof. In fact, it is well known (see, for instance, [2, p. 104]) that the algebra $\operatorname{sl}(\mathbb{O})$ satisfies the identity $h$.

Let $M$ be a Malcev algebra and $\mathcal{R}(M)$ be the universal multiplicative enveloping algebra of $M$. We recall (see [4, Definition 2.3]) that the algebra $\mathcal{R}(M)$ is defined up to isomorphism as the universal object with respect to representations of the algebra $M$. It can be constructed as the quotient algebra $T(M) / I$, where $T(M)$ is the tensor algebra of the vector space $M$ and $I$ is the ideal of $T(M)$ generated by the set $\{x \otimes y \otimes z-z \otimes x \otimes y-(y z) \otimes x-(x y) z+y \otimes(x z) \mid x, y, z \in M\}$ (see [4, p. 89]). An alternative way to construct $\mathcal{R}(M)$ is the following one. Consider the free product $M^{\prime}=M * F x$ of the algebra $M$ and the one-dimensional Malcev algebra $F x$ in the variety of Malcev algebras (see [1]); then the algebra $\mathcal{R}(M)$ is isomorphic to the subalgebra of the algebra $\operatorname{End}_{F}\left(M^{\prime}\right)$ generated by all the multiplication operators $R_{m}, m \in M$ (see [14).

We denote by $\mathcal{R}$ the linear mapping $\mathcal{R}: M \rightarrow \mathcal{R}(M)$ which maps $a \in M$ to $\mathcal{R}_{a}=a+I \in \mathcal{R}(M)$. For an ideal $K$ of $M$, we denote by $\mathcal{R}_{K}$ the ideal of the algebra $\mathcal{R}(M)$ generated by the set $\left\{\mathcal{R}_{x} \mid x \in K\right\}$.

Recall that in any Malcev algebra the following identity holds (see 9]):

$$
2 J(x, y, z) t=J(t, x y, z)+J(t, z x, y)+J(t, y z, x) .
$$

By $J(M)$ we denote the subspace of $M$ generated by all Jacobians $J(x, y, z)$, $x, y, z \in M$. It follows from (11) that $J(M)$ is an ideal of $M$.

Lemma 3. For an element $f \in J(M)$ and for an ideal $K$ of the algebra $M$, the inclusion $\left(\mathcal{R}_{f}\right)^{n} \in \mathcal{R}_{K}$ holds for some $n>0$ if and only if there exists an $m>0$ such that $M f^{m} \subseteq K$.

Proof. We note first that if $\left(\mathcal{R}_{f}\right)^{n} \in \mathcal{R}_{K}$ for some $n \in \mathbb{N}$, then obviously $M f^{n} \subseteq$ $M \mathcal{R}_{K} \subseteq M K \mathcal{R}(M) \subseteq K$.

Now assume that the inclusion $M f^{m} \subseteq K$ holds for some $m \in \mathbb{N}$. Let $f=$ $\sum_{i} J\left(a_{i}, b_{i}, c_{i}\right)$ for some $a_{i}, b_{i}, c_{i} \in M$. Then by (1) we have in the algebra $M^{\prime}=$ $M * F x$,

$$
2 x f=2 \sum_{i} x J\left(a_{i}, b_{i}, c_{i}\right)=\sum_{i} J\left(x, c_{i}, a_{i} b_{i}\right)+\sum_{i} J\left(x, a_{i}, b_{i} c_{i}\right)+\sum_{i} J\left(x, b_{i}, c_{i} a_{i}\right) .
$$

Furthermore, using identity (11) again, we have

$$
\begin{aligned}
4 x f^{2}= & \sum_{i} J\left(f, x c_{i}, a_{i} b_{i}\right)+\sum_{i} J\left(f, c_{i}\left(a_{i} b_{i}\right), x\right)+\sum_{i} J\left(f,\left(a_{i} b_{i}\right) x, c_{i}\right) \\
& +\sum_{i} J\left(f, x a_{i}, b_{i} c_{i}\right)+\sum_{i} J\left(f, a_{i}\left(b_{i} c_{i}\right), x\right)+\sum_{i} J\left(f,\left(b_{i} c_{i}\right) x, a_{i}\right) \\
& +\sum_{i} J\left(f, x b_{i}, c_{i} a_{i}\right)+\sum_{i} J\left(f, b_{i}\left(c_{i} a_{i}\right), x\right)+\sum_{i} J\left(f,\left(c_{i} a_{i}\right) x, b_{i}\right) .
\end{aligned}
$$


Now, by the defining Malcev identity,

$$
\begin{array}{rl}
4(-1)^{m} & x f^{m+2} \\
= & \sum_{i} J\left(f, x c_{i}, a_{i} b_{i} f^{m}\right)+\sum_{i} J\left(f, c_{i}\left(a_{i} b_{i}\right) f^{m}, x\right)+\sum_{i} J\left(f,\left(a_{i} b_{i}\right) x, c_{i} f^{m}\right) \\
& +\sum_{i} J\left(f, x a_{i}, b_{i} c_{i} f^{m}\right)+\sum_{i} J\left(f, a_{i}\left(b_{i} c_{i}\right) f^{m}, x\right)+\sum_{i} J\left(f,\left(b_{i} c_{i}\right) x, a_{i} f^{m}\right) \\
& +\sum_{i} J\left(f, x b_{i}, c_{i} a_{i} f^{m}\right)+\sum_{i} J\left(f, b_{i}\left(c_{i} a_{i}\right) f^{m}, x\right)+\sum_{i} J\left(f,\left(c_{i} a_{i}\right) x, b_{i} f^{m}\right) .
\end{array}
$$

Since by assumption $M f^{m} \subseteq K$, we have

$$
\begin{aligned}
x f^{m+2} & \in \sum_{i} J\left(f, x c_{i}, K\right)+\sum_{i} J\left(f, x a_{i}, K\right)+\sum_{i} J\left(f, x b_{i}, K\right)+J(f, K, x) \\
& +\sum_{i} J\left(f,\left(a_{i} b_{i}\right) x, K\right)+\sum_{i} J\left(f,\left(b_{i} c_{i}\right) x, K\right)+\sum_{i} J\left(f,\left(c_{i} a_{i}\right) x, K\right) \subseteq x \mathcal{R}_{K} .
\end{aligned}
$$

Clearly, this implies that $\mathcal{R}_{f}^{m+2} \in \mathcal{R}_{K}$.

Denote by $\mathbb{M}=\mathbb{M}(F)$ the split simple 7-dimensional Malcev algebra over the field $F$ (see [5, 6]) and by $T(\mathbb{M})$ the subset of the free Malcev algebra $\mathcal{M}_{\infty}$ on a countably infinite set of generators consisting on all stable identities of the algebra $\mathbb{M}$, that is, the identities all of whose partial linearizations are also identities of M. By the standard arguments (see, for example, [16, p. 317]), $T(\mathbb{M})$ is a $T$-ideal of $\mathcal{M}_{\infty}$; moreover, for every associative commutative $F$-algebra $K$, the algebra $\mathbb{M}(K)=K \otimes_{F} \mathbb{M}$ satisfies all identities from $T(\mathbb{M})$.

The following result follows easily from [2, Theorem 1] by the same arguments as for free alternative algebras in [10]. We give its proof for the sake of completeness.

Theorem 1. $\operatorname{rad} \mathcal{M}_{\infty}=J\left(\mathcal{M}_{\infty}\right) \cap T(\mathbb{M})$.

Proof. Let $f=f\left(x_{1}, \ldots, x_{n}\right) \in \operatorname{rad} \mathcal{M}_{\infty}$. The free Lie algebra $\mathcal{M}_{\infty} / J\left(\mathcal{M}_{\infty}\right)$ is evidently semiprime, which implies that $\operatorname{rad} \mathcal{M}_{\infty} \subseteq J\left(\mathcal{M}_{\infty}\right)$. In order to prove that $f \in T(\mathbb{M})$, it suffices to prove that $f$ is an identity in the algebra $\mathbb{M}(K)$, where $K$ is an infinite domain containing $F$. Take $K=F[t]$ and $\mathbb{M}_{1}=\mathbb{M}(F[t])$. Assume that $f$ is not the identity in $\mathbb{M}_{1}$. Then there exist elements $c_{1}, \ldots, c_{n} \in \mathbb{M}_{1}$ such that $f\left(c_{1}, \ldots, c_{n}\right) \neq 0$. Since $\mathbb{M}_{1}$ is finitely generated over $F$, there exists an epimorphism $\varphi: \mathcal{M}_{\infty} \rightarrow \mathbb{M}_{1}$ such that $\varphi\left(x_{i}\right)=c_{i}$. We have

$$
\operatorname{rad} \mathbb{M}_{1} \supseteq \varphi\left(\operatorname{rad} \mathcal{M}_{\infty}\right) \ni \varphi(f)=f\left(c_{1}, \ldots, c_{n}\right) \neq 0 .
$$

But the algebra $\mathbb{M}_{1}$ is a central order in the simple central 7-dimensional algebra $\mathbb{M}(F(t))$ over the field $F(t)$ and by 8 is prime. Thus $\operatorname{rad} \mathbb{M}_{1}=0$, a contradiction.

Conversely, let $f \in J\left(\mathcal{M}_{\infty}\right) \cap T(\mathbb{M})$. If $f \notin \operatorname{rad} \mathcal{M}_{\infty}$, then there exists a prime Malcev algebra $M$ and an epimorphism $\varphi: \mathcal{M}_{\infty} \rightarrow M$ such that $\varphi(f) \neq 0$. In other words, $f$ is not an identity in $M$. It is clear that $M$ is not a Lie algebra. By 2, Theorem 1], then $M$ is a central order in a simple 7-dimensional Malcev algebra $\widetilde{M}$ over an extension $K$ of $F$. Clearly, $f$ is not an identity of $\widetilde{M}$. Without loss of generality, we may assume that $K$ is algebraically closed and $\widetilde{M}$ is split. But then $\widetilde{M} \cong K \otimes_{F} \mathbb{M}$ and $\widetilde{M}$ satisfies all the identities from $T(\mathbb{M})$. Hence $f$ is an identity of $\widetilde{M}$, a contradiction. 
For an algebra $M$, denote by Engel $M$ the set of all universally engelian elements of $M$.

Theorem 2. Let $\mathcal{M}$ be the free Malcev algebra on more than two generators over a field $F$ of characteristic $\neq 2$. Then $\operatorname{rad} \mathcal{M}=$ Engel $\mathcal{M}$.

Proof. Let us first show that Engel $\mathcal{M} \subseteq \operatorname{rad} \mathcal{M}_{\infty}$. Clearly, every universally engelian element remains so under an epimorphism. Therefore, by Theorem 1, it suffices to show that the quotient algebras $\mathcal{M} / J(\mathcal{M})$ and $\mathcal{M} /(T(\mathbb{M}) \cap \mathcal{M})$ have no nonzero universally engelian elements. For the free Lie algebra $\mathcal{M} / J(\mathcal{M})$ it is clear. Let us prove this for the algebra $\mathcal{M} /(T(\mathbb{M}) \cap \mathcal{M})$. Let $f=f\left(x_{1}, \ldots, x_{n}\right)$ be a universally engelian element of $\mathcal{M} /(T(\mathbb{M}) \cap \mathcal{M})$. Then this algebra satisfies the identity $x_{n+1} f^{k}=0$. Observe that the algebra $\mathcal{M} /(T(\mathbb{M}) \cap \mathcal{M})$ is isomorphic to the free algebra (on the same number of generators) in the variety generated by the algebra $\mathbb{M}(F[t])$. In particular, the identity $x_{n+1} f^{k}=0$ is valid in $\mathbb{M}(K)$ for every extension $K$ of $F$. By [16, Theorem 2.8], there exists an extension $K$ of $F$ and a division octonion algebra $\mathbb{O}$ over $K$. Let $\widetilde{K}$ be the algebraic closure of $K$. Then the Malcev algebra $\widetilde{K} \otimes_{K} \operatorname{sl}(\mathbb{O})$ is split and therefore is isomorphic to $\mathbb{M}(\widetilde{K})$. Thus it satisfies the identity $x_{n+1} f^{k}=0$ and consequently so does the algebra $\operatorname{sl}(\mathbb{O})$. Assume that there exist $a_{1}, \ldots, a_{n} \in \operatorname{sl}(\mathbb{O})$ such that $a=f\left(a_{1}, \ldots, a_{n}\right) \neq 0$. Then $a$ is a nonzero engelian element in $\operatorname{sl}(\mathbb{O})$, which is impossible according to Corollary 1. This proves the inclusion Engel $\mathcal{M} \subseteq \operatorname{rad} \mathcal{M}_{\infty}$.

Now, we will prove that $\operatorname{rad} \mathcal{M} \subseteq$ Engel $\mathcal{M}$. Suppose conversely that there exists $f \in \operatorname{rad} \mathcal{M}$ which is not universally engelian in $\mathcal{M}$. Consider the set of ideals $\Sigma=\left\{K \triangleleft \mathcal{M} \mid \forall m \in \mathbb{N}, \mathcal{R}_{f}^{m} \notin \mathcal{R}_{K}\right\}$. The set $\Sigma$ is not empty because $\{0\} \in \Sigma$; in addition $\Sigma$ is partially ordered by inclusions. If $\left\{K_{s} \mid s \in S\right\}$ is a chain of elements of $\Sigma$, then $\bigcup_{s \in S} K_{s} \in \Sigma$. By the Zorn lemma, $\Sigma$ has a maximal element $P$. We will prove that $P$ is a prime ideal in $\mathcal{M}$. Suppose that $K, L \triangleleft \mathcal{M}$ such that $K \supseteq P$, $L \supseteq P$, and $K L \subseteq P$. By hypothesis, there exist $l_{1} \in \mathbb{N}$ and $l_{2} \in \mathbb{N}$ such that $\mathcal{R}_{f}^{l_{1}} \in \mathcal{R}_{K}$ and $\mathcal{R}_{f}^{l_{2}} \in \mathcal{R}_{L}$. Then $\mathcal{R}_{f}^{l_{1}+l_{2}} \in \mathcal{R}_{K} \mathcal{R}_{L}$, and we have

$$
\mathcal{M} f^{l_{1}+l_{2}} \subseteq K f^{l_{2}} \subseteq(K L) \mathcal{R}(\mathcal{M}) \subseteq P .
$$

Since $f \in J(\mathcal{M})$, by Lemma 3 we have $\mathcal{R}_{f}^{m} \subseteq \mathcal{R}_{P}$ for some $m$, a contradiction. Therefore the ideal $P$ is prime and $f \notin P$, which is impossible.

We have proved that $\operatorname{rad} \mathcal{M} \subseteq$ Engel $\mathcal{M} \subseteq \operatorname{rad} \mathcal{M}_{\infty} \cap \mathcal{M}$. But if $A$ is a subalgebra of $B$, then $(\operatorname{rad} B) \cap A \subseteq \operatorname{rad} A$. Therefore, $\operatorname{rad} \mathcal{M}=\operatorname{rad} \mathcal{M}_{\infty} \cap \mathcal{M}=$ Engel $\mathcal{M}$.

The theorem is proved.

Observe that we have also proved the following result of independent interest:

Corollary 2. For every $n>2 \operatorname{rad} \mathcal{M}_{n}=\operatorname{rad} \mathcal{M}_{\infty} \cap \mathcal{M}_{n}=T(\mathbb{M}) \cap J\left(\mathcal{M}_{n}\right)$.

We finish the paper with two open questions:

Problem 1. Is $\operatorname{rad} \mathcal{M}_{\infty}\left(\operatorname{rad} \mathcal{M}_{n}\right)$ nilpotent?

Problem 2. Is $\operatorname{rad} \mathcal{M}_{4} \neq 0$ ?

Observe that recently the second author has proved that $\operatorname{rad} \mathcal{M}_{3}=0$. The proof will appear elsewhere. In [12, 13, it was proved that the radical of a free alternative algebra is nilpotent if the set of generators is finite or the base field has characteristic 0 . This easily implies that, under the same conditions, the answer to the first question is positive if the algebra $\mathcal{M}_{\infty}$ is special, that is, can be embedded into the commutator algebra of an alternative algebra. 


\section{ACKNOWLEDGEMENT}

The authors thank the referee for useful suggestions and remarks.

\section{REFERENCES}

[1] P. M. Cohn, Universal Algebra, Harper \& Row Publishers, New York-London, 1965. MR0175948 (31:224)

[2] V. T. Filippov, On the theory of $\mathrm{Mal}^{\prime} \mathrm{cev}$ algebras (Russian), Algebra $i$ Logika, 16, no. 1 (1977), 101-108. MR0506526 (58:22219)

[3] V.T. Filippov, Nilpotent ideals in $\mathrm{Mal}^{\prime} \mathrm{cev}$ algebras (Russian), Algebra i Logika, 18, no. 5 (1979), 599-613. MR582105 (82b:17024)

[4] N. Jacobson, Structure and Representations of Jordan Algebras, AMS Colloq. Publ. 39, AMS, Providence, RI, 1968. MR0251099 (40:4330)

[5] E. N. Kuz'min, Mal'cev algebras and their representations (Russian), Algebra i Logika 7, no. 4 (1968), 48-69; English transl. in Algebra and Logic 7, no. 4 (1968), 233-244. MR0252468 (40:5688)

[6] E. N. Kuz'min and I.P. Shestakov, Nonassociative structures (Russian), Current problems in mathematics. Fundamental directions, Vol. 57, 179-266, Itogi Nauki i Tekhniki, Akad. Nauk SSSR, Vsesoyuz. Inst. Nauchn. i Tekhn. Inform., Moscow, 1990; English transl. in Encyclopaedia of Math. Sciences 57, Algebra VI, eds. A.I. Kostrikin and I.R. Shafarevich. Springer-Verlag, 1995, 199-280. MR.1060322 (91i:17001)

[7] A.I. Mal'cev, Analytic loops, Mat. Sb. (N.S.) 36 (78) (1955), 569-575. MR0069190 $(16: 997 \mathrm{~g})$

[8] S. V.Polikarpov and I. P. Shestakov, Nonassociative affine algebras (Russian), Algebra $i$ Logika, 29, no. 6 (1990), 709-723; English transl. in Algebra and Logic 29, no. 6 (1990), 458-466. MR1159142 (93b:17009)

[9] A. A. Sagle, Malcev algebras, Trans. Amer. Math. Soc. 101, no. 3 (1961), 426-458. MR0143791 (26:1343)

[10] I. P. Shestakov, The radical and nilpotent elements of free alternative algebras (Russian), Algebra i Logika, 14, no. 3 (1975), 354-365; English transl. in Algebra and Logic, 14, no. 3 (1975), 219-226. MR 0427413 (55:447)

[11] I. P. Shestakov, On a problem by Shirshov (Russian), Algebra i Logika, 16, no. 2 (1977), 227246; English transl. in Algebra and Logic, 16, no. 2 (1977), 153-166. MR516039 (81c:17023)

[12] I. P. Shestakov, Finitely generated special Jordan and alternative PI-algebras (Russian), Mat. Sb. (N.S.) 122 (164) (1983), no. 1, 31-40. MR715833 (84k:17018)

[13] I. P. Shestakov and E. I. Zelmanov, Prime alternative superalgebras and nilpotence of the radical of a free alternative algebra, Izv. Akad. Nauk SSSR Ser. Mat. 54, no. 4 (1990), 676-693. MR1073082 (91j:17003)

[14] Ivan Shestakov and Natalia Zhukavets, The universal multiplicative envelope of the free Malcev superalgebra on one odd generator, Communications in Algebra 34, no. 4 (2006), 1319-1344. MR2220815 (2007b:17051)

[15] E. I. Zel'manov, Primary Jordan algebras (Russian), Algebra i Logika 18, no. 2 (1979), 162175. MR566779 (81k:17012)

[16] K. A. Zhevlakov, A. M. Slin'ko, I. P. Shestakov, and A. I. Shirshov, Rings that are nearly associative (Russian), Moscow, Nauka, 1978; English translation by Academic Press, 1982, N.Y. MR518614 (80h:17002)

Institute of Mathematics and Statistics, University of São Paulo, Rua do Matao, 1010, Cidade Universitária, São Paulo 05508-090, Brazil

imeCC Cidade Universitária Zeferino Vaz, Campinas, 13083-859 SÃo Paulo, Brazil

Current address: Centro de Matemática, Computação e Cognição, Universidade Federal do ABC, Rua Santa Adélia, 166, Blocoa, Bairro Bangu, Santo André, SP, Brazil 09210-170 